\title{
Sensorimotor synchronization with chords containing tone-onset asynchronies
}

\author{
Michael J. Hove \\ Cornell University, Ithaca, New York \\ Peter E. Keller \\ Max Planck Institute for Human Cognitive and Brain Sciences, Leipzig, Germany \\ AND \\ Carol L. KrumhansL \\ Cornell University, Ithaca, New York
}

\begin{abstract}
Musical ensemble performance requires the synchronization of multiple performers, resulting in sequences of chords containing multiple tones with multiple onsets. Experiments 1 and 2 investigate whether sensorimotor synchronization with chord sequences containing tone-onset asynchronies is affected by (1) the magnitude of these asynchronies $(25,30$, or $50 \mathrm{msec})$ and (2) the pitch of the leading tone (high vs. low). Participants tapped a finger in synchrony with different types of chord sequences created by crossing these variables, as well as with sequences of chords containing simultaneous onsets. Results indicate that taps were drawn toward the second onset, when present, especially when it was lower in pitch than the first. Additionally, chords with nonsimultaneous onsets increased tapping variability for nonmusicians, but decreased variability for musicians. Experiment 3 measured the perceptual centers of the chords from Experiment 2, and yielded results suggesting that subjective onsets determine the temporal placement of taps during synchronization.
\end{abstract}

The synchronization of rhythmic movements with auditory sequences is a key aspect of skilled musical performance. Musicians routinely synchronize their actions with chords containing multiple component tones. For example, a soloist must synchronize with chords of a piano accompaniment, or with different voices in an orchestra or jazz rhythm section. In the context of live human performance, the onsets of these multiple components are typically not in perfect temporal synchrony. The experiments reported here investigate the effect that asynchronous tone onsets have on tap timing accuracy and variability during sensorimotor synchronization.

Asynchronous onsets of musical tones are common and arise from various sources. At the most basic level, asynchronies reflect limitations in synchronization accuracy that stem from perceptual, cognitive, and motor implementation constraints. Due to these limitations, consistently perfect synchrony is not possible. Musical training, however, greatly decreases error and variability in synchronization (e.g., Gérard \& Rosenfeld, 1995; Pressing \& Jolley-Rogers, 1997; Repp \& Penel, 2002).

Nevertheless, highly trained expert musicians often intentionally produce asynchronous onsets between nominally simultaneous notes in both solo and ensemble music. Analyses of timing in solo piano performance re- veal that the melody note often precedes other voices by 20-50 msec, thereby increasing the perceptual salience of the melody line (Palmer, 1996); or it may be, at least in part, a velocity artifact of louder dynamics (Goebl, 2001; Repp, 1996). Some pianists systematically lead with low tones, and this represents a deliberate, expressive strategy (Repp, 1996). Asynchronies produced for expressive purposes are also common in ensemble performance. For example, Rasch (1988) examined the respective note onsets of string trio players and found the violin's lead voice preceded the cello by $5-10 \mathrm{msec}$ on average, which preceded the viola by another 5-10 msec. Purposeful and systematic onset asynchronies have also been studied in the context of jazz and popular music, where they are thought to be a key factor in the somewhat nebulous musical concepts of "groove," "feel," and "swing" (Collier \& Collier, 1997; Friberg \& Sundström, 2002; Iyer, 2002; Keil, 1995). Timing analyses of an Afro-Cuban percussion ensemble showed that the lead drum tended to play $30 \mathrm{msec}$ ahead of the clave, a reference percussion instrument (Bilmes, 1993). Other examples include the bass lead in James Brown's band, the "wide beat" of the Miles Davis Quintet of the 1960s, the RZA's nonquantized hiphop grooves, and even some of the orchestral works of Brahms. ${ }^{1}$ 
Despite their widespread presence in music, onset asynchronies are often not consciously perceived by listeners. A wide pitch difference between asynchronous tones, and different timbres and rise times, contribute to onset asynchronies passing unnoticed. In addition, attention may be drawn more to the "horizontal" timing of one melody line than to the "vertical" relations between simultaneous lines (Rasch, 1988).

However, psychophysical experiments have shown that onset asynchronies are easily perceived under carefully controlled laboratory conditions. Approximately $20 \mathrm{msec}$ is sufficient to discern the temporal order of two asynchronous sounds for highly trained listeners (Hirsh, 1959); untrained listeners may need much longer time intervals (Broadbent \& Ladefoged, 1959). In sensorimotor synchronization experiments, displacing a pacing sequence event by less than $20 \mathrm{msec}$ from its putatively expected time may not be consciously detected in perception, but nevertheless may elicit compensatory changes in tap timing (Repp, 2001, 2002). Larger values can affect the perceptual organization of musical textures. Specifically, it has been shown that tone sequences temporally offset by about $30 \mathrm{msec}$ or more tend to be perceived as separate streams, if the frequency difference between tones is sufficiently large (Bregman \& Pinker, 1978).

The existence of systematic onset asynchronies in music, together with the results from the psychophysical literature, raise the question of whether these asynchronies affect sensorimotor synchronization with the perceived beat (i.e., the most salient level of pulsation experienced when listening to music). Specifically, is the temporal goal for synchronization based solely on the first onset within a chord complex, on the average of all onsets, or on some other form of temporal derivative such as the perceptual center (P. G. Vos, Mates, \& van Kruysbergen, 1995) of the chord? Furthermore, given that the presence of multiple tone onsets within a chord creates the potential for multiple synchronization targets, is movement timing variability increased under such circumstances?

The literature on sensorimotor synchronization dates back over 120 years (for a recent review, see Repp, 2005). Experiments generally consist of synchronizing a movement, often a finger tap, with an external metronome. Typical dependent variables in such experiments are mean tap-to-metronome asynchrony and mean intertap interval (ITI) and the variability of both measures. Recently, researchers have started to investigate sensorimotor synchronization with more musically relevant stimuli (Large, 2000; Repp, 1999; Snyder \& Krumhansl, 2001). Nevertheless, studies that address issues related to the effects of onset asynchronies on sensorimotor synchronization remain rare, with the exception of recent work by Repp (2003, 2004).

Repp (2003, 2004) instructed his participants to tap in synchrony with the tones of a "target" sequence while ignoring an interleaved "distractor" sequence that either lagged behind or anticipated the target sequence by varying amounts. The target sequence was presented for a while before its accompanying distractor sequence began. Target and distractor sequences differed in pitch by 3-20 semi- tones, and ranged from $1976 \mathrm{~Hz}$ to $4192 \mathrm{~Hz}$, depending on experiment. Repp $(2003,2004)$ found that participants had difficulty ignoring the distractors when they occurred within 100-150 msec of the targets. Such distractors drew taps away from the target sequence, particularly when they preceded the target tones, and increased tap timing variability. The effects of the distractors being higher or lower in pitch than the targets were inconsistent, and for a few reasons it is unclear how these findings relate to the effects of onset asynchronies in musical performance. First, in most instances of ensemble performance, musicians are required to attend to other instrumental parts rather than to ignore them. Second, the target and distractor tones used by Repp $(2003,2004)$ were high in frequency and out of the typical pitch range of rhythm-setting musical instruments.

This article presents three experiments. Experiments 1 and 2 investigate whether sensorimotor synchronization with chord sequences containing tone-onset asynchronies is affected by (1) the magnitude of these asynchronies and (2) the pitch of the leading tone. Control sequences composed of chords with simultaneously sounding tone onsets were also employed. To simulate partially the demands of synchronization during musical ensemble performance, participants were simply instructed to synchronize "with the beat" rather than with just one specific component of the chord, and the lowest tone $(350 \mathrm{~Hz})$ in the chords was close to the center of the typical musical pitch range. The effects of musical experience on synchronization under these conditions were assessed by testing people with various amounts of musical training. Note that tone-onset asynchrony was held constant from chord to chord within a sequence, whereas in musical performance asynchronies naturally fluctuate from chord to chord. We opted to hold tone-onset asynchrony constant for reasons of experimental control; otherwise, chord sequences with simultaneous and nonsimultaneous onsets would vary not only in terms of relative tone-onset times, but also in terms of whether onset times varied from chord to chord within a sequence. Experiment 3 examines the perceived onset time, or perceptual center (P-center), of chords containing onset asynchronies in order to assess whether tap timing in the first two experiments was based on P-centers rather than on physical event onsets.

\section{EXPERIMENT 1}

The purpose of Experiment 1 was to investigate whether tone-onset asynchronies affect sensorimotor synchronization. Toward this end, a sequence of two-tone chords was presented in which a low tone slightly preceded a high tone. In a control condition, two-tone chords in which the onsets were synchronous were used. Four dependent measures were used as indices of performance accuracy: mean tap-to-chord asynchronies, variability (standard deviation) of asynchronies within a trial, mean ITIs, and within-trial ITI variability. For simplicity in analysis, the first tone onset of a chord was considered to be the chord onset. Indeed, if a chord is perceived as a single event despite the presence of onset asynchronies, the onset of the first tone 
is the physical onset of this event. Asynchronies are each based on the timing of a single tap relative to an event in the pacing signal, whereas ITIs are based on the timing of two adjacent taps independently of the pacing signal. Thus, asynchronies provide an index of the phase relationship between taps and the pacing sequence, whereas ITIs are a measure of tapping period, or tempo.

\section{Method}

Participants. Fifteen adult, unpaid volunteers (10 women, 5 men; age range, 22-33 years; mean age, 27.1), including authors M.J.H. and P.E.K., participated in Experiment 1. All participants were right-handed, as assessed by self-report. Total years of musical performance experience ranged from 0 to 20 years $(M=8.2$ years). Eight participants, hereafter referred to as "musicians," had more than 6 years of formal musical training and ensemble experience. The remaining participants were designated "nonmusicians."

Materials. The chords used in the stimulus sequences were composed of two sine tones: a short $50-\mathrm{msec}$ high tone $(1400 \mathrm{~Hz})$ and a slightly longer $80-\mathrm{msec}$ low tone $(350 \mathrm{~Hz})$. The high tone was 2 octaves above the low tone. The tones were generated with CoolEdit software, equated for loudness, and were combined in two different types of chords: (1) the low-tone onset preceded the high-tone onset by $30 \mathrm{msec}$, so the tones ended together; or (2) both tone onsets were presented simultaneously, so the 80 -msec low tone ended $30 \mathrm{msec}$ after the 50-msec high tone. This resulted in chords with two segments, one in which only a single tone sounded, and another in which two tones sounded. After superimposing the high and low tones, the amplitude of the single-tone segment (at either the onset or the offset) was adjusted so that it and the two-tone segment had equal maximum amplitude levels $(60 \mathrm{~dB}$ under our presentation conditions). Thus, the two segments of the chord differed in frequency content, but not in amplitude. This amplitude equalization allowed investigation of effects of pitch on synchronization while minimizing any confounding effect of different amplitude. Despite being less natural, the amplitude change within the longer tone was imperceptible and the chords still sounded pleasant and musical to authors M.J.H. and P.E.K. Each trial consisted of a single chord presented 20 times successively with a stimulus interonset interval (IOI) of $500 \mathrm{msec}$. The $500-\mathrm{msec}$ IOI reflects the time between the first occurring element in one chord (whether the low tone or simultaneous low + high tone) and the first element of the next chord.

Procedure. Participants sat in front of a computer monitor that displayed the trial number, and listened to the sequences over PRO-10 isolation headphones. They tapped with their right index fingers on a $3 \times 3 \mathrm{~cm}$ metal plate that produced no sound audible through the headphones. The sequence was presented and taps were recorded via Experimental Run Time System (accurate to $1 \mathrm{msec}$ ).

Participants were instructed to start tapping with the 3rd chord and to tap in synchrony with "the beat." The presence of high and low tones and their onset/offset difference was not mentioned in the instructions. The experiment consisted of 4 blocks of 12 randomized trials ( 6 of each of the two chord types per block). The first 2 trials of each block (one for each chord type) were not analyzed, so of the 48 total trials, 40 were analyzed. Trials were initiated by the participants, and short breaks were included after each block. The entire experiment lasted approximately $20 \mathrm{~min}$.

\section{Results and Discussion}

It typically took a few cycles for synchronization to stabilize at the beginning of a trial, and therefore only taps corresponding to chords 7-19 (taps 5-17) were analyzed; our analysis therefore focused exclusively on asymptotic performance from the trials. Within-trial standard deviations of tap-to-chord asynchronies were screened for outliers. Of the 600 total trials, 9 trials yielded greater than
2.5 times an individual's average standard deviation and were excluded from the analyses.

Average data for mean asynchronies, $S D$ of asynchronies, mean ITIs, and $S D$ of ITIs are shown in Table 1. These data were entered into separate ANOVAs with the within-participants factor chord type (simultaneous vs. nonsimultaneous) and the between-participants factor musical experience (musician vs. nonmusician). The criterion for statistical significance was set at $\alpha=.05$ for all analyses reported in this article. ${ }^{2}$

The tap-to-chord asynchrony was measured from the onset of the first tone (or tones) in such a way that taps that preceded tones resulted in negative asynchrony values. The asynchronies were compared for the two trial types. The anticipation tendency common in finger tapping experiments (e.g., Aschersleben, 2002) was observed; taps tended to precede chord onsets. Moreover, our analysis revealed that mean tap-to-chord asynchrony for chords containing simultaneous onset of high and low tones was significantly larger (i.e., more negative) than for chords in which the high-tone onset was delayed $30 \mathrm{msec}$ relative to the low-tone onset $[F(1,13)=6.02, p<.05]$. In other words, the taps tended to occur slightly $(\sim 4 \mathrm{msec})$ later, and nearer to the physical onset of the chord, when the high-tone onset followed the low-tone onset. Neither musical experience nor the interaction of chord type and musical experience affected mean asynchrony reliably $(p \mathrm{~s}>.6)$.

Although the placement of taps relative to events in the pacing sequence was affected by chord type, tapping rate was relatively immune to effects of this variable. In fact, participants were, on the whole, highly successful at tapping at the correct tempo: Mean ITI (500.02 msec) was close to the target ITI $(500 \mathrm{msec})$, and was not affected reliably by chord type or musical training or by their interaction $(p s>.1)$.

The ANOVAs on our two indices of tap timing variability ( $S D$ of asynchronies and $S D$ of ITIs) revealed comparable results. First, there was no significant main effect of chord type on either measure ( $p s>.6)$. Second, tap timing variability was generally lower in musicians than in nonmusicians: Average within-trial $S D$ of asynchronies

Table 1

Mean Asynchrony, Within-Trial Standard Deviations (SDs) of Asynchronies, Mean Intertap Intervals (ITIs), and Within-Trial SDs of ITIs Averaged Across Musicians and Nonmusicians From Experiment 1

\begin{tabular}{lcc}
\hline & \multicolumn{2}{c}{ Chord Type } \\
\cline { 2 - 3 } \multicolumn{1}{c}{ Measure } & Simultaneous Onset & Nonsimultaneous Onset \\
\hline Mean asynchrony & -44.35 & -40.77 \\
& $(36.03)$ & $(32.48)$ \\
SD of asynchronies & 19.98 & 20.06 \\
& $(3.77)$ & $(4.06)$ \\
Mean ITI & 499.83 & 500.20 \\
& $(0.88)$ & $(0.92)$ \\
SD of ITIs & 24.60 & 24.47 \\
& $(4.39)$ & $(5.70)$ \\
\hline
\end{tabular}

Note-The between-participants $S D$ of scores on each measure is shown in parentheses. All values are in milliseconds. 
was $17.81 \mathrm{msec}$ for musicians and $22.58 \mathrm{msec}$ for nonmusicians $[F(1,13)=9.25, p<.01]$; average $S D$ of ITIs was $21.24 \mathrm{msec}$ for musicians and $28.31 \mathrm{msec}$ for nonmusicians $[F(1,13)=16.23, p=.001]$. Finally, and most interestingly, there was a significant interaction between chord type and musical experience on both measures: $S D$ of asynchronies $[F(1,13)=5.51, p<.05]$; $S D$ of ITIs $[F(1,13)=5.34, p<.05]$. The results of separate comparisons for musicians and nonmusicians were clearest for the $S D$ of ITIs measure, an index of ability to maintain a steady tempo. As shown in Figure 1, the $S D$ of ITIs was lower with nonsimultaneous onsets than with simultaneous onsets for musicians $[t(7)=2.40, p<.05]$, whereas results are numerically in the opposite direction for nonmusicians, though this effect was not reliable $[t(6)=$ $-1.24, p>.2]$.

Overall, the results of this experiment indicate that chords containing tone-onset asynchronies affected tap timing; in particular, taps were drawn toward the second onset. However, the shift was only $\sim 4 \mathrm{msec}$, not the 15 -msec shift one might expect if the mean of the two tone onsets (30-msec delay in individual tone onsets) were considered the chord's "onset." This suggests that tap timing is determined predominantly by the first onset within a chord containing onset asynchronies. Perhaps surprisingly, tapping variability was generally not degraded by the presence of onset asynchronies in the pacing sequence; on the contrary, participants with musical training tapped with less variability when the chords contained nonsimultaneous onsets.

\section{EXPERIMENT 2}

The purpose of Experiment 2 was to investigate in more detail the effects on sensorimotor synchronization of the magnitude of tone-onset asynchrony in chords (none, $25 \mathrm{msec}, 50 \mathrm{msec}$ ) and the pitch of the leading tone (high tone first or low tone first). Thus, participants were required to tap in synchrony with five different types of sequence. Dependent measures were again tap-to-chord

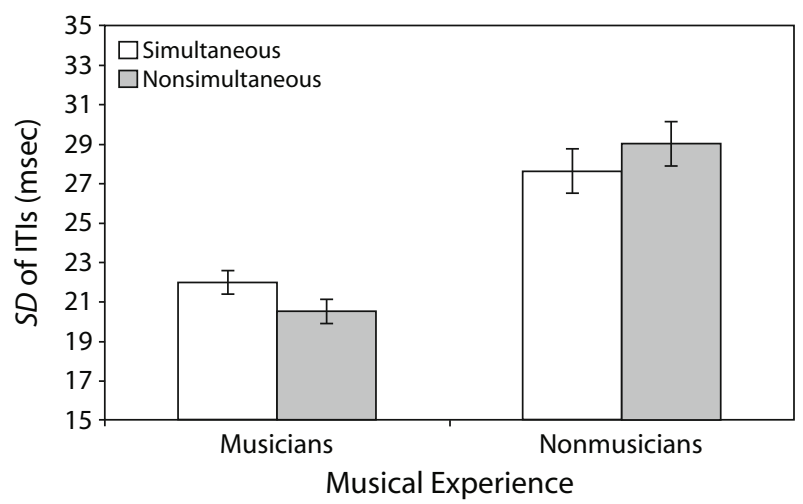

Figure 1. Average within-trial SD of ITIs with simultaneous and nonsimultaneous onset chords for musicians and nonmusicians in Experiment 1. Error bars represent $95 \%$ confidence intervals calculated separately for musicians and nonmusicians (see Masson \& Loftus, 2003). asynchrony (as measured from the first tone onset), the $S D$ of these asynchronies, mean ITI, and the $S D$ of ITIs. On the basis of the results of Experiment 1, we expected that mean asynchrony would be shifted toward the second onset of chords containing onset asynchronies. Whether the amount of shift would be affected by magnitude of tone-onset asynchrony, and whether synchronization would be affected by the order in which the high and low tones occurred within a chord, were questions of interest.

\section{Method}

Participants. Seventeen adults (12 women, 5 men; age range, 19-31 years; mean age, 24.8) participated in the experiment. ${ }^{3}$ Most were students from Ludwig Maximilian University, Munich. All participants were right-handed, as assessed by self-report. Total musical performance experience ranged from 0 to 20 years $(M=$ 10 years). Seven participants ("musicians") had more than 6 years of formal musical training and ensemble experience. Participants were previously unfamiliar with the task, and were paid $€ 8$ each for participating.

Materials. As in Experiment 1, each stimulus sequence consisted of chords composed of two sine tones - a high tone $(1400 \mathrm{~Hz})$ and a low tone $(350 \mathrm{~Hz})$ generated beforehand in CoolEdit and equated for loudness. The tones were combined in five different types of chords: (1) the high-tone onset was followed by the low-tone onset after $50 \mathrm{msec}$ (hereafter denoted as H-50-L); (2) the high-tone onset was followed by the low-tone onset after $25 \mathrm{msec}(\mathrm{H}-25-\mathrm{L})$; (3) the high tone and low tone were presented simultaneously (simultaneous); (4) the low-tone onset was followed by the high-tone onset by $25 \mathrm{msec}(\mathrm{L}-25-\mathrm{H})$; and (5) the low-tone onset was followed by the high-tone onset by $50 \mathrm{msec}(\mathrm{L}-50-\mathrm{H})$. Tone offsets were simultaneous for all chords, and occurred $100 \mathrm{msec}$ after the onset of the first tone. As before, in order to examine the effects of pitch without confounding amplitude information, the amplitude (dB levels) of the individual tone component was adjusted so that it and the chord component had equal maximum $\mathrm{dB}$ levels.

Procedure. The procedure was identical to that in Experiment 1, with the following exceptions: Each trial consisted of a single chord presented 21 times successively, with an IOI of $550 \mathrm{msec}$. Participants were instructed to start tapping with the fifth chord in each trial. Taps 5-16 (corresponding to chords 9-20) were analyzed. There were 8 blocks of 20 trials (plus one training block). Four trials from each of the 5 conditions appeared in each block, with presentation order randomized. Participants each took approximately $1 \mathrm{~h}$ to complete the experiment.

\section{Results and Discussion}

Data were again screened for outliers. Of the 2,720 total trials, 62 had standard deviations of tap-to-chord asynchronies greater than $60 \mathrm{msec}$ ( $\sim 2.5$ times overall average $S D$ ) and were excluded. Additionally, individual tap-totone asynchronies were examined for anomalies. Participants occasionally stopped tapping a few beats early and would subsequently try to resynchronize, resulting in deviant asynchronies in 57 trials. These individual asynchronies were not included in the analyses reported below (165 total deleted data points from the 57 trials).

Mean asynchronies in each of the five chord type conditions are shown in Figure 2 (averaged across musical experience). These data were entered into a $5 \times 2$ ANOVA with the within-participants factor chord type (L-50-H, L-25-H, simultaneous, H-25-L, H-50-L) and the betweenparticipants factor musical experience (musician vs. nonmusician). This ANOVA revealed a statistically signifi- 
cant effect of chord type on mean asynchrony $[F(4,60)=$ 23.67, $p<.001$ ] (the Greenhouse-Geisser correction was applied here and in all cases below when the degrees of freedom numerator exceeded 1). Neither the main effect of musical experience nor the interaction between chord type and musical experience was statistically significant $(p \mathrm{~s}>.3)$.

The effect of chord type on mean asynchrony was unpacked using four planned orthogonal contrasts (with data collapsed across musical experience). The first contrast compared asynchronies in the chord type condition with simultaneous tone onsets (simultaneous condition) with asynchronies in the remaining conditions combined (i.e., averaged across the H-50-L, H-25-L, L-25-H, and L-50-H conditions). This contrast revealed that taps in the simultaneous condition occurred significantly earlier than taps to chords containing tone-onset asynchronies $[F(1,16)=$ $33.4, p<.001]$. This result was expected and replicates the main result of Experiment 1. The second contrast examined the magnitude of tone-onset asynchrony (50 msec vs. $25 \mathrm{msec}$ ). Participants tapped significantly later for chords containing the larger tone-onset asynchronies $[F(1,16)=28.4, p<.001]$. Thus, the amount of delay before the second tone onset affected tap timing. The third contrast examined the effect of the leading pitch (hightone lead vs. low-tone lead). Participants tapped significantly later for chords where the high tone led $[F(1,16)=$ $13.5, p<.01]$. It appears that low tones tend to attract taps more than high tones. The fourth contrast examined the interaction effect of high-/low-tone lead $\times 25 / 50$-msec onset delay and found a significant difference $[F(1,16)=$ 17.09, $p<.001]$. This interaction can be seen in Figure 2 as a greater difference between the effect of tone-onset delay when the high tone led.

A $5 \times 2$ ANOVA on mean ITIs revealed that participants were, on the whole, able to tap at the correct tempo: Mean ITI was $549.73 \mathrm{msec}$ (the target ITI was $550 \mathrm{msec}$ ) and was not affected reliably by chord type, musical training, or by their interaction $(p s>.4)$.

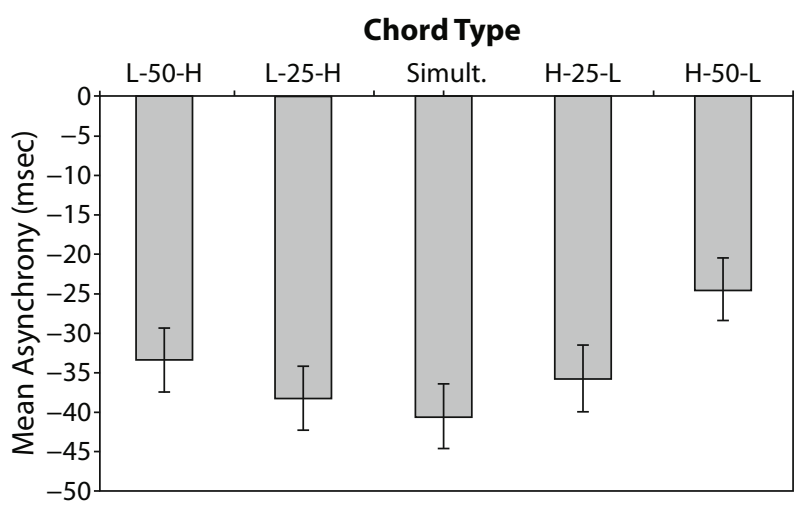

Figure 2. Average tap-to-chord asynchronies in Experiment 2 for the five different chord types (e.g., "Simult." chords have simultaneous onsets of high and low tones and in "H-50-L" chords, the high-tone onset precedes the low-tone onset by $50 \mathrm{msec}$ ). The error bars represent standard error.
Table 2

Within-Trial Standard Deviation (SD) of Asynchronies and Within-Trial $S D$ of Intertap Intervals (ITIs) Averaged Across Musicians and Nonmusicians From Experiment 2 Chord Type

\begin{tabular}{lccccc}
\cline { 2 - 6 } \multicolumn{1}{c}{ Measure } & L-50-H & L-25-H & Simult. & H-25-L & H-50-L \\
\hline$S D$ of asynchronies & 20.52 & 20.97 & 21.07 & 21.05 & 21.46 \\
& $(5.58)$ & $(5.22)$ & $(5.42)$ & $(5.19)$ & $(4.93)$ \\
$S D$ of ITIs & 24.38 & 24.90 & 25.08 & 25.35 & 25.97 \\
& $(6.06)$ & $(5.75)$ & $(5.89)$ & $(6.22)$ & $(5.95)$ \\
\hline
\end{tabular}

Note-The between-participants $S D$ of scores on each measure is shown in parentheses. All values are in milliseconds. L-50-H, low-tone onset followed by high-tone onset by $50 \mathrm{msec}$; L-25-H, low-tone onset followed by high-tone onset by $25 \mathrm{msec}$; Simult., simultaneous onset; H25-L, high-tone onset followed by low-tone onset by $25 \mathrm{msec} ; \mathrm{H}-50-\mathrm{L}$, high-tone onset followed by low-tone onset by $50 \mathrm{msec}$.

We turn now to our indices of tap timing variability. Averaged data for $S D$ of asynchronies and $S D$ of ITIs are shown in Table 2. A $5 \times 2$ ANOVA on $S D$ of asynchronies revealed no significant main effects of chord type or musical experience, and no significant interaction effect for these factors ( $p$ s $>.1)$. However, the corresponding ANOVA on $S D$ of ITIs revealed a significant effect of chord type $[F(4,60)=3.02, p<.05]$. The main effect of musical experience was not significant $(p>.9)$, and the interaction between chord type and musical experience approached significance $[F(4,60)=2.22, p=.09]$. A follow-up analysis employing the same four planned orthogonal contrasts described above (with musical experience included as a between-participants factor) revealed that the effect of chord type was confined to the contrast that tested effects of leading tone pitch: $S D$ of ITIs was lower on average when the low tone led than when the high tone led $[F(1,15)=5.59, p<.05]$. Chords in which the onset of the low tone preceded the onset of the high tone apparently facilitated tapping regularity.

Our contrast analysis also revealed a significant interaction between chord type and musical experience on the contrast that compared SD of ITIs for chords with simultaneous versus nonsimultaneous onsets $[F(1,15)=16.42$, $p<.001]$. This effect gives weight to the results of Experiment 1 . As can be seen in Figure 3, musicians' tapping was more stable in the nonsimultaneous conditions, on average, than in the simultaneous condition $[t(6)=3.55, p=$ .01]. Nonmusicians, conversely, tapped more stably in the simultaneous condition $[t(9)=-2.53, p<.05]$. Musical training did not produce a significant interaction with chord type on the remaining three contrasts $\left[F_{\mathrm{S}}(1,15)<1\right]$.

\section{EXPERIMENT 3}

The mean asynchrony results of Experiments 1 and 2 raise the question why people tap later with chords containing onset asynchronies. Here we investigated the potential role of perceptual centers in this effect. P-centersthe subjective moments of occurrence of an acoustic signal-have been studied widely in both speech and musical contexts (e.g., Gordon, 1987; Morton, Marcus, \& Frankish, 1976; Scott, 1998; J. Vos \& Rasch, 1981). P. G. 


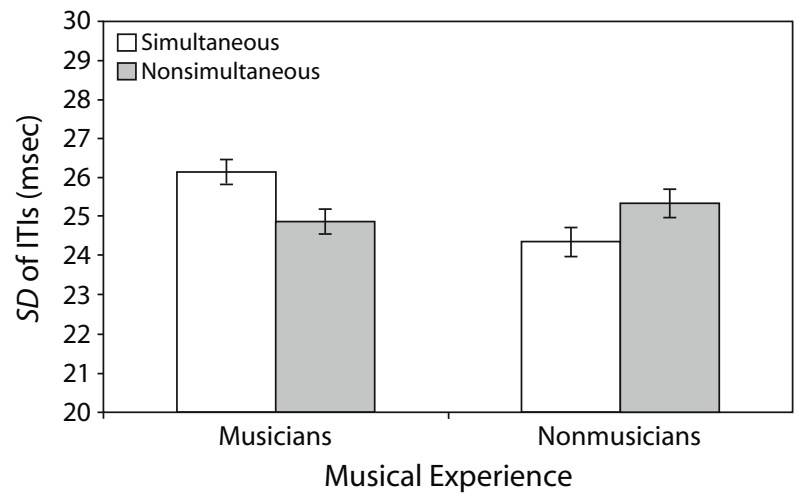

Figure 3. Average within-trial $S D$ of ITIs with simultaneous and nonsimultaneous onset chords for musicians and nonmusicians in Experiment 2. Error bars represent $\mathbf{9 5 \%}$ confidence intervals calculated separately for musicians and nonmusicians.

Vos et al. (1995) manipulated the duration and rise time of single-tone stimuli in a series of tapping experiments and demonstrated that, as predicted by the P-center hypothesis, asynchronies became less negative, with longer stimulus durations and less steep rise times. One potential explanation for the results of Experiment 1 and 2 is that participants attempted to align their taps with the P-center of the chord, and that a chord's P-center was later when one of its constituent tones had a delayed onset. Moreover, to account for the results of Experiment 2, the P-center would need to be later when the low-tone onset followed the high-tone onset rather than the reverse. Experiment 3 was designed to test this hypothesis by measuring the P-center of the five chord types from Experiment 2. The P-center-focused explanation of the effects of onset asynchrony on synchronization would be supported by finding that the relative positioning of P-centers across the five chord types is similar to the tap positioning reflected in the mean asynchrony results from Experiment 2.

\section{Method}

Participants. Twelve Cornell University undergraduates participated (3 women and 9 men; age range 18-23 years, mean age, 20.2). All participants had some musical training, ranging from 3 to 12 years of instruction $(M=7.0)$. Six "musicians" had more than 6 years of formal training. Participants received course credit for their participation, and were previously unfamiliar with the task.
Materials and Procedure. The same five chords as in Experiment 2 were used. Participants sat in front of a Macintosh laptop and listened to the tones over AKG K141 headphones. Tones were presented and data were recorded using a program written in MAX.

Participants initiated each trial with a mouse click, which in turn triggered two interleaved metronomes, both with IOIs of 1,000 $\mathrm{msec}$, as shown in Figure 4.

The constant "reference" metronome started; then, after a delay, so did the adjustable "target" metronome. The initial delay of the target metronome was randomly selected from a range of 300-700 msec with a step size of $1 \mathrm{msec}$.

Participants were instructed to adjust the target metronome so that its onsets bisected the reference metronome onsets. The target metronome onsets could be shifted by clicking one of 4 buttons: a large left arrow, a small left arrow, a small right arrow, and a large right arrow. These keys had the effect of shifting the target metronome $15 \mathrm{msec}$ earlier, $1 \mathrm{msec}$ earlier, $1 \mathrm{msec}$ later, and $15 \mathrm{msec}$ later, respectively. When participants felt that the onsets of the two metronomes were equally spaced in time, they could click a button labeled "Just Right" and commence the next trial.

Reference metronome tones were always the simultaneous chords from Experiment 2, in which the high and low tones were presented simultaneously. The target metronome tones were one of the five chord types from Experiment 2 (H-50-L, H-25-L, simultaneous, L-25-H, L-50-H). The five trial types were presented 10 times each for a total of 50 trials in the experiment. Participants took between 45 and 70 min to complete the experiment. ${ }^{4}$

\section{Results and Discussion}

The P-center for each chord type was calculated by measuring its mean adjusted onset time after the preceding reference metronome onset and then subtracting 500 from this value. For example, the target metronome onset (i.e., the onset of the first tone) for $\mathrm{H}-50$-L chords was, on average, $479 \mathrm{msec}$ after the reference metronome onset, so its perceived onset occurred $21 \mathrm{msec}$ after the actual onset of the first tone of the chord in the target metronome.

Average P-centers for the five chord types are shown in Figure 5. Note the qualitative resemblance between the pattern of results in this graph and the mean asynchrony results from Experiment 2 (Figure 2). A $5 \times 2$ ANOVA with the within-participants factor chord type and the between-participants factor musical experience (musician vs. nonmusician) on the P-center data revealed neither an effect of musical experience nor an interaction $(p$ s > .3). However, the ANOVA revealed a significant effect of chord type $[F(4,36)=12.10, p<.001]$. Borrowing the logic used in Experiment 2, this effect was unpacked using four planned orthogonal contrasts (with data

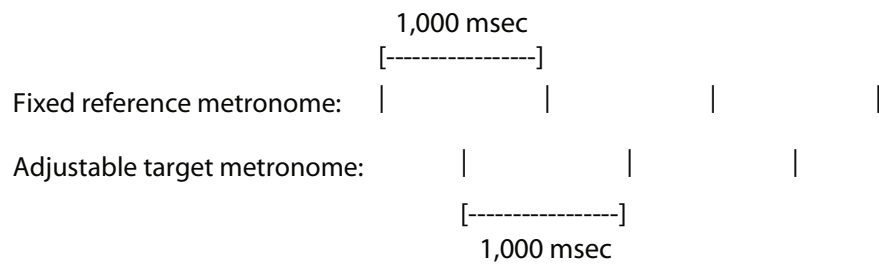

Figure 4. Graphical depiction of the interleaved metronomes in Experiment 3. Between 300 and $700 \mathrm{msec}$ after a fixed reference metronome commenced, an adjustable "target" metronome started. Participants adjusted the phase of the target metronome until they felt that the two metronomes' respective onsets bisected each other. 


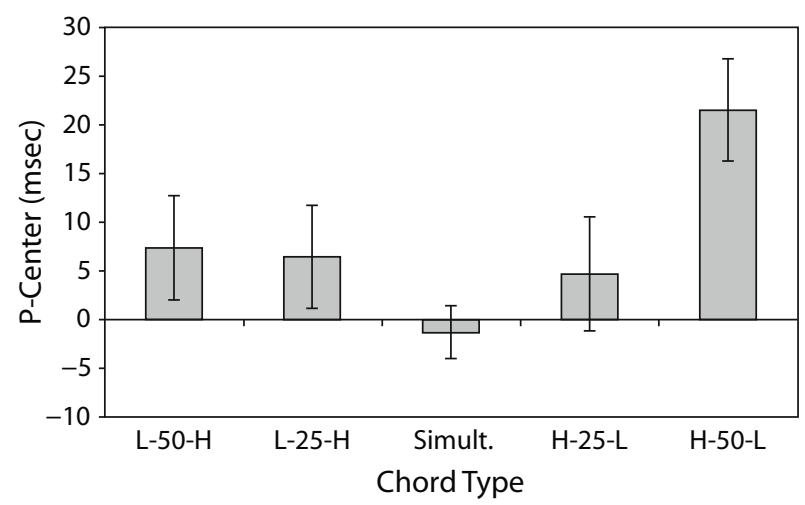

Figure 5. Average P-centers for the five chord types, as measured relative to the onset of the chord's first tone in Experiment 3. For example, the $\mathbf{H}-\mathbf{5 0}-\mathrm{L}$ chord's $\mathrm{P}$-center is $21 \mathrm{msec}$ after its initial tone onset. The error bars represent standard error.

collapsed across musical experience). The first planned contrast revealed that the P-center was later for chords containing tone-onset asynchronies than for chords with simultaneous tone onsets $[F(1,10)=11.23, p<.01]$. Second, the P-center was, on average, later for chords with large tone-onset asynchronies than for small onset asynchronies $[F(1,10)=29.40, p<.001]$. Third, the P-center was, on average, earlier for chords in which the low tone led than when the high tone led $[F(1,10)=6.62, p<$ $.05]$. This effect was largely due to a very late P-center for chords where the high tone led by $50 \mathrm{msec}$, as evidenced by a significant interaction between the pitch of the leading tone and the magnitude of the tone-onset asynchrony $[F(1,10)=8.98, p<.02]$. Thus, the results of the statistical analysis are qualitatively the same as those observed for mean asynchrony in Experiment 2. P-centers seem to go quite some way in explaining the earlier results; the question is, can they go all the way?

To test the degree to which P-centers accounted for the effects of chord type on mean asynchronies in Experiment 2, we entered the pooled data from Experiments 2 and 3 into a $5 \times 2$ ANOVA with the within-participants factor chord type and the between-participants factor experiment. Not surprisingly, this ANOVA revealed a significant main effect of chord type, which simply reflects the fact that chord type affected both tap placement and P-centers $[F(4,104)=32.47, p<.001]$. A significant main effect of experiment was also observed $[F(1,26)=$ $18.69, p<.001]$ : Taps (recorded in Experiment 2) preceded P-centers (measured in Experiment 3 ) by $42.8 \mathrm{msec}$ on average. However, the interaction between chord type and experiment was not significant $[F(4,104)=1.36, p>$ $.2]$. This suggests that the relative positioning of taps across the chord-type conditions can be accounted for largely by differences in P-centers between the chord types.

\section{GENERAL DISCUSSION}

The three experiments reported here were motivated by the observation that nonsimultaneous tone onsets occur commonly in polyphonic music such as solo piano perfor- mance (e.g., Palmer, 1996; Repp, 1996) and in ensemble performance (Prögler, 1995; Rasch, 1988). Our main question was whether such tone-onset asynchronies affected sensorimotor synchronization. The first two experiments examined how tone-onset asynchronies within the chords of a pacing sequence affected tap timing accuracy (i.e., tap placement relative to chord onsets) and variability (ability to maintain a constant phase relationship and tapping tempo). The final experiment investigated whether the observed effects could be attributed to shifts in the perceptual onsets (the P-centers) of the chords.

\section{Tap Timing Accuracy}

In the first two experiments, we obtained the common result that taps precede stimulus onsets by $25-50 \mathrm{msec}$. These negative mean asynchronies support the idea that, in sensorimotor synchronization, physical onsets of external events (taps and tone onsets) are not synchronized; rather, synchrony is established on a central level of subjective simultaneity (Aschersleben, 2002), so that the P-centers of taps and tones are aligned. Because processing delays are longer in the tactile than in the auditory modality, taps must occur "early" for perceptual co-occurrence.

Negative asynchronies were found for two-tone chords with both simultaneous and nonsimultaneous onsets in Experiments 1 and 2, but the magnitude of the asynchrony varied. Our results indicate that asynchronies between taps and initial chord onsets shifted toward the onset of a delayed second tone and that the shift was larger when the second tone onset was delayed longer. However, the magnitude of shift was much less than half the difference between the first and second tone onsets. For example, chords containing a 25 -msec onset delay had average tap shifts of approximately 3 and $5 \mathrm{msec}$ (depending on whether the high tone preceded the low tone or vice versa, as discussed below) rather than of $12.5 \mathrm{msec}$, and chords containing a 50-msec onset delay had average tap shifts of 7 and $15 \mathrm{msec}$ rather than of $25 \mathrm{msec}$. Thus, the first tone onset of a chord has a larger influence on determining tap timing than a subsequent onset in the chord. Presumably, the first onset is more perceptually salient. ${ }^{5}$

Results from Experiment 3 showed similar shifts in a chord's P-center as a function of onset delay of one component. The magnitude of $\mathrm{P}$-center shift was again smaller than the mean onset difference. For chords with a $25-\mathrm{msec}$ onset asynchrony, we saw shifts in P-center of 6 and $8 \mathrm{msec}$ relative to the simultaneous-condition P-center (depending on whether the high or low tone led); for chords with a 50 -msec delay, we observed shifts of 9 and $22 \mathrm{msec}$. The fact that these effects are smaller than half the difference between the tone onsets supports the idea that the first tone onset is more important in determining the group's P-center. Thus, the effects of nonsimultaneous tone onsets within a chord are somewhat analogous to the effects of lengthening a sound's duration or decreasing the steepness of its rise time.

Pooled data from Experiments 2 and 3 revealed that a chord's P-center largely accounts for tap timing when that chord is used in a pacing sequence. As stated above, we assume that participants attempt to line up P-centers 
for taps and tones. The perceived onsets of taps (i.e., P-centers established on the basis of efferent and afferent movement-related information) are presumably relatively constant across our conditions. So when the P-center for tones changes as a function of chord type, tap timing must change in order to realign the tap P-centers with the new tone P-centers. When the tone P-centers occur later than the P-centers of chords with simultaneous tone onsets, taps must be delayed so that their P-centers are aligned with the tone P-centers.

Our findings are consistent with previous work that has shown that when multiple tones sound within a perceptual temporal integration window of approximately 100 $150 \mathrm{msec}$, the tones are perceived as a tightly bound group rather than as separate events (Repp, 2005; Yabe et al., 1998). In a related study mentioned briefly in the introduction, Repp (2003, Experiment 2) asked participants to synchronize tapping with a target sequence and to ignore a distractor sequence of a different pitch in which tones slightly preceded or followed tones in the target sequence. Participants' tap timing inadvertently shifted more toward early distractors than late distractors. For example, when distractors preceded targets by $40 \mathrm{msec}$, taps shifted approximately $30 \mathrm{msec}$ earlier, whereas when distractors followed targets by $40 \mathrm{msec}$, taps shifted approximately $8 \mathrm{msec}$ later. These results align nicely with our findings, namely that multiple onsets occurring close together are perceived as an integrated unit and subsequent tap timing is dictated by a weighted average of component onsets with more "weight" placed on the first onset.

We observed pitch differences indicating that low tones had a greater effect on tapping. The anticipation tendency is smaller for chords with delayed low tones, because the $\mathrm{P}$-center is later for these chords. Additionally, the ability to maintain a steady tapping tempo was best for chords in which the onset of the low tone preceded the onset of the high tone. In the Repp (2003) study mentioned above, a few weak effects of pitch on tap timing were observed; participants could synchronize with low-pitch targets and ignore high-pitch distractors better than vice versa. These results are consistent with our finding that lower pitched tones attract taps more than higher pitched tones when both high and low pitched tones are present. In a related experiment, Handel and Lawson (1983) presented participants with polyrhythms (e.g., 2:3, 3:4, 4:5, and 3:4:5) consisting of different pitched pulse trains, and examined rhythmic interpretations via participants' preferred tapping patterns. Participants tended to select the lowest pitched pulse train as the polyrhythm meter. A possibility to consider is that low tones mask delayed high tones more than vice versa because of the "upward spread of masking" (Moore, 1997). A decrease in onset salience of high tones that follow low tones might result in the high tones' relatively small impact on P-center judgments. It should be noted, however, that in the present experiments the high tones were easily audible so only partial masking may have occurred, if any.

Finally, the results may be related to the musical convention that often assigns the role of setting the beat to low-pitched instruments (e.g., walking bass lines in jazz, tuba in oompah bands, the left hand in ragtime piano, "four on the floor" bass drum in some electronic music). Our participants may have been influenced by this musical convention, or the convention itself may be rooted in the greater perceptual salience of lower tones. Additionally, there may be a contribution of a cross-modal system that favors a connection between low tones and physical movements. Indeed, the low, loud sounds encountered in techno dance music have been shown to evoke a vestibular response that may be pleasurable and may induce movement (Todd, 2001; Todd \& Cody, 2000).

\section{Tap Timing Variability}

Our two synchronization experiments also revealed some effects of the type of chord onset (simultaneous or nonsimultaneous) on the variability of tap timing. These effects were subtle (it should be noted that data from trials with abnormally high variability were excluded from our analyses). The most revealing measure of tapping variability was the $S D$ of the ITIs, which is an index of how well participants were able to tap at a steady tempo. The effect of chord onset asynchronies on this measure depended on musical experience. Chords with nonsimultaneous onsets tended to decrease tap regularity for participants with little or no musical training. This is what would generally be expected if the nonsimultaneous onsets introduced uncertainties in either perception or how the task was to be performed. In contrast, nonsimultaneous onsets actually facilitated tap regularity for participants with considerable musical training. We will briefly explore a few factors potentially contributing to this effect. Note that the mechanisms invoked by these explanations may operate independently of those that determine P-centers.

Facilitation of tapping regularity would result from increased constancy of motor command pulses established by auditory input. Although slightly counterintuitive, multiple proximal onsets might actually increase the precision of temporal pulse in later stages of processing. Each temporal event has an associated variance, and, assuming additivity of variance, the $S D$ of the weighted average of multiple onsets will be less than that of single onsets. If musicians could efficiently integrate nonsimultaneous onsets, this variance would decrease.

Alternatively, increased tapping regularity might result if nonsimultaneous onsets rendered error correction mechanisms less active. Numerous synchronization models (e.g., Mates, 1994a, 1994b; Pressing, 1998; Semjen, Schulze, \& Vorberg, 2000; Vorberg \& Wing, 1996) propose that taps are adjusted by a proportion of recent synchronization error in order to mitigate drift; however, the adjustments have an associated "cost" of increased ITI variability (Semjen et al., 2000; Voillaume, 1971). A number of researchers (e.g., Mates, 1994a; Schulze \& Vorberg, 2002) suggest that the asynchrony must exceed a certain threshold before error correction mechanisms are engaged, although most existing error correction models do not include such a threshold, an exception being Mates's (1994a, 1994b) model. ${ }^{6}$ In the present context, nonsimultaneous onsets may have enlarged the span of time over which deviations are too small to engage error 
correction mechanisms. In other words, when there are two onsets, the likelihood of "being on the beat" increases, so phase correction responses would be less active and ITI variance would decline.

A final explanation of why onset asynchrony might reduce tapping variability for some participants has to do with dynamical systems models, self-sustained internal oscillators, and entrainment (Large \& Jones, 1999; Large \& Palmer, 2002). According to Large and Jones's (1999) model of perception and attention, an adjustable oscillating "attentional pulse" reacts only to onsets that occur close to the time at which they are expected to occur; if the metronome onset does not occur at the peak of the internal oscillator's temporal cycle but is still within the range of the attentional pulse, a readjustment of the internal oscillator's phase is caused. If the oscillator and external beats frequently coincide, the region of sensitivity will shrink; conversely, if internal and external oscillations are more variable, the region will widen. Of greatest relevance to the present results, Large and Palmer (2002) showed that the model was better at tracking temporal changes in a pulse when the pulse was articulated by chords with multiple onsets. In other words, two onsets will increase the likelihood of "being on the beat." Although these models are of perception-attention coupling, "being more on the beat" might improve perception-action coupling as previously mentioned. Another possibility is that, although the peaks are wider, they are more regularly spaced, which could facilitate tapping stability. Finally, nonsimultaneous onset chords might represent a stronger dynamical attractor, because there is more perceptual information to lock onto. Whereas existing models of synchronization may not directly address our manipulations of asynchrony or the importance of pitch, extensions thereof could.

\section{Conclusions}

The main outcome of the current study is that sensorimotor synchronization is affected markedly by tone-onset asynchronies that fall within the range of asynchronies observed in multipart music performance. Our results suggest that such onset asynchronies affect the P-centers of chords in the pacing sequence thereby influencing the location of the temporal target for synchronization. This effect of onset asynchronies on tap placement appears to be independent of musical experience, implying that its source lies in a natural synergy between perception and action during synchronization.

On the other hand, more subtle effects of onset asynchronies on the ability to maintain a steady tapping tempo appear to be modulated by musical experience. Individuals with large amounts of musical performance experience, in contrast to individuals with little or no such experience, displayed greater tempo stability when onset asynchronies were present than when such asynchronies were absent. Although this finding may seem surprising at first, it can be accounted for by a combination of various existing theoretical assumptions; furthermore, it falls well within the realm of musical intuition. Musical experts should deal better than novices with structural ambiguity in a pacing sequence. Years of exposure to nonsimultaneous tone on- sets might lead to the avoidance of any potential conflict by allowing musicians to perceive an integrated "beat" under such circumstances, whereas nonmusicians might be confused by onset asynchronies, potentially leading to the perception of multiple displaced beats.

However, far from merely dealing relatively well with structural ambiguity, musicians may in fact thrive on it. Musicians debate whether it is better to play in strict simultaneity or slightly out of phase (Keil, 1995). The present work might offer this insight: The beat you play depends on the audience. When playing for other musicians, nonsimultaneity or a "wide beat" will result in more regular movements (dance steps, head bobs, finger snaps), and will, therefore, arguably be more enjoyable. On the other hand, nonmusicians move more regularly with "unambiguous" synchronous onsets, hence, such simultaneity may be preferred. This difference in beat preference is reflected in the music industry. Much popular music today is quantized, and this absence of microtiming deviations may appeal to many of the musically untrained consumers. Conversely, musical training might increase appreciation of expressive microtiming deviations and onset asynchronies that are common in live groove-based music.

\section{AUTHOR NOTE}

This research was supported by the Max Planck Society and Cornell University. We thank Kerstin Traeger and Joyce Rosenbaum for assisting with data collection, and Mari Riess Jones, Edward Large, Bruno Repp, and an anonymous reviewer for helpful comments on an earlier version of the manuscript. Correspondence concerning this article should be addressed to M. J. Hove, B93 Uris Hall, Cornell University, Ithaca, NY 14853-7601 (e-mail: mjh88@cornell.edu).

\section{REFERENCES}

Aschersleben, G. (2002). Temporal control of movements in sensorimotor synchronization. Brain \& Cognition, 48, 66-79.

Bellman, J. (2003). Performing Brahms in the style hongrois. In M. Musgrave \& B. D. Sherman (Eds.), Performing Brahms: Early evidence of performance style (pp. 327-349). Cambridge: Cambridge University Press.

Bilmes, J. A. (1993). Timing is of the essence: Perceptual and computational techniques for representing, learning, and reproducing expressive timing in percussive rhythm. Unpublished masters' thesis, Massachusetts Institute of Technology.

Bregman, A. S., \& Pinker, S. (1978). Auditory streaming and the building of timbre. Canadian Journal of Psychology, 32, 19-31.

Broadbent, D. E., \& LAdefoged, P. (1959). Auditory perception of temporal order. Journal of the Acoustical Society of America, 31, 1539.

Collier, G. L., \& Collier, J. L. (1997). The microstructure of jazz rhythm. In H. Martin (Ed.), Annual review of jazz studies (Vol. 8, pp. 117-140). Lanham, MD: Scarecrow Press.

FriberG, A., \& Sundström, A. (2002). Swing ratios and ensemble timing in jazz performance: Evidence for a common rhythmic pattern. Music Perception, 19, 333-349.

GÉrARd, C., \& Rosenfeld, M. (1995). Pratique musicale et régulations temporelles [Musical expertise and temporal regulation]. L'Année Psychologique, 95, 571-591

GoebL, W. (2001). Melody lead in piano performance: Expressive device or artifact? Journal of the Acoustical Society of America, 110, 563-572.

Gordon, J. W. (1987). The perceptual attack time of musical tones. Journal of the Acoustical Society of America, 82, 88-105.

Handel, S., \& Lawson, G. R. (1983). The contextual nature of rhythmic interpretation. Perception \& Psychophysics, 34, 103-120.

Hirsh, I. J. (1959). Auditory perception of temporal order. Journal of the Acoustical Society of America, 31, 759-767. 
IYER, V. (2002). Embodied mind, situated cognition, and expressive microtiming in African-American music. Music Perception, 19, 387-414.

KeIL, C. (1995). The theory of participatory discrepancies: A progress report. Ethnomusicology, 39, 1-19.

Large, E. W. (2000). On synchronizing movements to music. Human Movement Science, 19, 527-566.

Large, E. W., \& Jones, M. R. (1999). The dynamics of attending: How we track time-varying events. Psychological Review, 106, 119-159.

Large, E. W., \& Palmer, C. (2002). Perceiving temporal regularity in music. Cognitive Science, 26, 1-37.

Masson, M. E. J., \& LofTus, G. R. (2003). Using confidence intervals for graphically based data interpretation. Canadian Journal of Experimental Psychology, 57, 203-220.

MATES, J. (1994a). A model of synchronization of motor acts to a stimulus sequence: I. Timing and error corrections. Biological Cybernetics, 70, 463-473.

MATES, J. (1994b). A model of synchronization of motor acts to a stimulus sequence: II. Stability analysis, error estimation and simulations. Biological Cybernetics, 70, 475-484.

Moore, B. C. J. (1997). An introduction to the psychology of hearing. San Diego: Academic Press.

Morton, J., Marcus, S., \& Frankish, C. (1976). Perceptual centers (P-centers). Psychological Review, 83, 405-408.

Palmer, C. (1996). On the assignment of structure in music performance. Music Perception, 14, 23-56.

PRESSING, J. (1998). Error correction processes in temporal pattern production. Journal of Mathematical Psychology, 42, 63-101.

Pressing, J., \& Jolley-Rogers, G. (1997). Spectral properties of human cognition and skill. Biological Cybernetics, 76, 339-347.

PRöGLER, J. A. (1995). Searching for swing: Participatory discrepancies in the jazz rhythm section. Ethnomusicology, 39, 21-54.

RASCH, R. A. (1988). Timing and synchronization in ensemble performance. In J. A. Sloboda (Ed.), Generative processes in music: The psychology of performance, improvisation, and composition (pp. 7090). Oxford: Oxford University Press, Clarendon Press.

RePP, B. H. (1996). Patterns of note onset asynchronies in expressive piano performance. Journal of the Acoustical Society of America, 100, 3917-3932.

RePP, B. H. (1999). Control of expressive and metronomic timing in pianists. Journal of Motor Behavior, 31, 145-164.

REPP, B. H. (2001). Phase correction, phase resetting, and phase shifts after subliminal timing perturbations in sensorimotor synchronization. Journal of Experimental Psychology: Human Perception \& Performance, 27, 600-621.

REPP, B. H. (2002). Automaticity and voluntary control of phase correction following event onset shifts in sensorimotor synchronization. Journal of Experimental Psychology: Human Perception \& Performance, 28, 410-430.

RePP, B. H. (2003). Phase attraction in sensorimotor synchronization with auditory sequences: Effects of single and periodic distractors on synchronization accuracy. Journal of Experimental Psychology: Human Perception \& Performance, 29, 290-309.

REPP, B. H. (2004). On the nature of phase attraction in sensorimotor synchronization with interleaved auditory sequences. Human Movement Science, 23, 389-413.

Repp, B. H. (2005). Sensorimotor synchronization: A review of the tapping literature. Psychonomic Bulletin \& Review, 12, 969-992.

Repr, B. H., \& Penel, A. (2002). Auditory dominance in temporal processing: New evidence from synchronization with simultaneous visual and auditory sequences. Journal of Experimental Psychology: Human Perception \& Performance, 28, 1085-1099.
Schulze, H.-H., \& Vorberg, D. (2002). Linear phase correction models for synchronization: Parameter identification and estimation of parameters. Brain \& Cognition, 48, 80-97.

ScotT, S. K. (1998). The point of P-centres. Psychological Research, 61, 4-11.

Semjen, A., Schulze, H.-H., \& Vorberg, D. (2000). Timing precision in continuation and synchronization tapping. Psychological Research, 63, 137-147

Snyder, J., \& Krumhansl, C. L. (2001). Tapping to ragtime: Cues to pulse finding. Music Perception, 18, 455-489.

ToDD, N. P. M. (2001). Evidence for a behavioral significance of saccular acoustic sensitivity in humans. Journal of the Acoustical Society of America, 110, 380-390.

TodD, N. P. M., \& Cody, F. W. (2000). Vestibular responses to loud dance music: A physiological basis of the "rock and roll threshold"? Journal of the Acoustical Society of America, 107, 496-500.

Voillaume, C. (1971). Modèles pour l'étude de la régulation des mouvements cadencés [Models for the study of cadenced movement regulation]. L'Année Psychologique, 71, 347-358.

VorBERG, D., \& Wing, A. (1996). Modeling variability and dependence in timing. In H. Heuer \& S. W. Keele (Eds.), Handbook of perception and action (Vol. 2, pp. 181-262). San Diego: Academic Press.

Vos, J., \& RASCH, R. (1981). The perceptual onset of musical tones. Perception \& Psychophysics, 29, 323-335.

Vos, P. G., Mates, J., \& van Kruysbergen, N. W. (1995). The perceptual centre of a stimulus as the cue for synchronization to a metronome: Evidence from asynchronies. Quarterly Journal of Experimental Psychology, 48A, 1024-1040.

Yabe, H., Tervaniemi, M., SinkKonen, J., Huotilainen, M., IlmoniEMI, R. J., \& NÄÄTÄNEN, R. (1998). Temporal window of integration of auditory information in the human brain. Psychophysiology, 35, 615-619.

\section{NOTES}

1. For example, in the first movement of Brahms's Piano Concerto No. 1, the composer enhances the salience of a structurally important location in the music by requiring the basses to sound on the final beat of one bar (i.e., an upbeat), followed by a dissonant chord in the rest of the ensemble on the first beat of the next bar. This ensemble bass lead reflects Brahms's expressive left hand lead, heard in the Edison cylinder recording of his piano performance of the Hungarian Dances (Bellman, 2003).

2. Analyses on linear statistics and circular statistics yielded the same pattern of results; therefore, only linear statistics are reported.

3. Data from an 18th participant could not be analyzed because he did not complete the full experimental procedure.

4. Data from one nonmusician (male, age 20) were not analyzed, because more than half of his trials were not recorded due to a technical error.

5. P. G. Vos et al. (1995) found evidence for the perceptual dominance of onsets in single tones of various durations. Taps were shifted toward the physical center of longer tones, but the physical onset was clearly a greater determinant of tap timing, since taps did not coincide with the midpoints of the tones.

6. Schulze and Vorberg (2002) demonstrated the difficulty of trying to detect a threshold empirically and showed that linear correction parameters can provide an excellent approximation "before more realistic but complex models arrive."

(Manuscript received November 23, 2005; revision accepted for publication October 31, 2006.) 